\title{
Side effects of pain and analgesia in animal experimentation
}

\author{
Paulin Jirkof
}

\author{
This review highlights selected effects of untreated pain and of widely used analgesics such as \\ opioids, non-steroid anti-inflammatory drugs and antipyretics, to illustrate the relevance of carefully \\ planned, appropriate and controlled analgesia for greater reproducibility in animal experiments \\ involving laboratory rodents.
}

A potential cause of suffering in experimental animals is pain induced by procedures, diseases and injuries. Pain is a complex state arising from many sources, involving several systems in the body. Pain is more than nociception and has been defined as a "subjective, sensory and emotional experience" 1 in man. It can be assumed that pain, as an affective experience, also exists in other mammals. In light of these considerations, pain treatment becomes an ethical, and in most countries legal, obligation in any animal experiment that induces more than mild pain of short duration.

Nevertheless, pain management is more than an animal welfare concern, as it has important scientific and methodological implications for the design of experiments and the quality of the resulting data. A recent review on reporting practices for anesthesia and analgesia protocols after invasive animal procedures revealed that many published studies do not include pain relief and do not report, or do not completely report, the anesthetic and analgesic measures involved ${ }^{2}$. The pain management protocol chosen, as well as any untreated pain, has the potential to affect scientific results and increase the variability of data, thereby hampering the reproducibility of experiments substantially. Pain management includes the choice of anesthesia and analgesia agents, their dose, administration method, duration and frequency of treatment, and a painmonitoring scheme for each individual animal. It is therefore an important part of experimental design, and determining the appropriate protocol is mandatory when planning animal experiments.

As a complement to such important preparatory work, this review aims to highlight some effects of untreated pain, as well as side effects of widely used analgesics, to illustrate the relevance of appropriate and controlled analgesia, as well as the proper reporting of all analgesic measures ${ }^{2}$ for greater reproducibility in animal experiments. As mice and rats are the most abundant laboratory species, this article focuses on these rodent species.

\section{Effects of untreated pain}

The sensory component of pain is nociception, which depends on specifically dedicated receptors, nociceptors, and associated pathways. Different brain structures and a network of neurons with a complex pattern of connections are then involved in the processing of pain and the resulting responses. Although some components of the system mediate sensory discriminant aspects, others convey information about affective-motivational aspects such as the unpleasantness that accompanies painful stimuli ${ }^{3}$. When tissue is traumatized, the release of local chemical agents such as inflammatory mediators may also cause excitation of nerve endings. This can lead to hyperalgesia, a state in which stimuli that are normally perceived as slightly painful are perceived as substantially more painful. A consequence of nerve damage can be allodynia, an increase in the excitability of neurons, which results in pain following a stimulus that is not normally perceived to be painful ${ }^{3}$.

Untreated pain may hamper the healing of tissues. Bone healing, for example, is promoted by mechanical stimulation. Thus, as has been shown in rats, effective pain relief and the consequent increased use of the affected body part are important for healing 4 .

Besides local mechanisms, ascending painful impulses lead to hypothalamic activation and increased sympathetic-adrenergic system activity. This activation results in substantial cardiovascular effects, such as changes in heart rate and heart rate variability, as has been shown in mice ${ }^{5}$. Pain, similar to distress, affects the secretion of many hormones, neurotransmitters and enzymes. For example, untreated pain increases the secretion of catecholamines such as noradrenalin, corticoids, glucagon, adrenocorticotrophic hormone $(\mathrm{ACTH})$ and antidiuretic hormone $(\mathrm{ADH})$, and decreases the secretion of insulin and testosterone in many species $^{6,7}$. In addition, untreated pain can affect the immune system detrimentally by leading to a reduction of natural killer cells and mixed lymphocyte reactivity ${ }^{8}$.

In humans, unrelieved pain may contribute to psychological distress, sleeplessness and impaired rehabilitation. Changes in behavior that might be indicative of negative affective states are also observed in animals. Typical changes in rodent behavior include reduced food and water intake, changes in activity, reduced sleep, a flattened circadian rhythm, loss of behavioral diversity, and changes in social grooming, nest building or burrowing behavior ${ }^{7,9-11}$. 
This exemplary list illustrates how pain affects a multitude of body systems via several pathways. Pain states following surgical trauma and painful diseases are complex in nature and cannot be easily predicted or controlled. Omitting pain relief to reduce side effects of analgesic drugs might be a double-edged sword, and is justifiable only in a few well-founded cases.

\section{Effect of analgesia protocols}

The choice of analgesia protocol for a specific research question is challenging and might best be solved with the help of an expert in veterinary analgesia. The optimal analgesia protocol should relieve pain reliably and lack side effects that might hamper animal welfare. In addition, it should have a controllable effect on the specific system targeted by the experiment or on experimental procedures. In light of the many aspects that have to be considered-species, type of pain, research question - there is no one-size-fits-all analgesia.

Pain is, in many cases, never relieved completely, but should be minimized and controlled. To choose a potent analgesia protocol, one must identify the type of pain (nociceptive, neuropathic, idiopathic or mixed), as well as its intensity and duration. There are distinct differences in the features of acute and chronic pain, and acute pain, if not well managed, can lead to chronic pain that persists even if the underlying cause has been treated. Although the course of pain in spontaneously developing diseases such as cancer may differ substantially in the individual, in most rodent surgeries pain peaks around the first $4-6 \mathrm{~h}$ post operation. Although the duration of pain depends on the effect of the surgery, even routine interventions, such as embryo transfer involving a minor laparotomy, can result in up to $24 \mathrm{~h}$ of moderate-to-mild pain ${ }^{5,10}$. Furthermore, pain perception might differ between species, between lines and strains, and between sexes, and also depends on the age of the animal ${ }^{12-15}$. In addition, possible sex differences in drug biotransformation and pharmacokinetics have been discussed ${ }^{16}$.

The pharmacokinetics of analgesics vary with the route of administration and analgesic drugs differ in their duration of action and therefore in the need for repeated administration. Finding the correct frequency of drug administration, which depends on the onset and duration of analgesic effect, is important for assuring constant pain relief. Failure to achieve an adequate and sustained drug level in the organism may lead to gaps in analgesia that represent animal welfare issues and might contribute to variability in data.

The choice of administration route and interval affects animal well-being as well as experimental schedule and read-out and should be planned out carefully. Furthermore, repeated injections or oral gavage of drugs require restraint of the animal, which can cause additional stress ${ }^{17}$ and might increase existing pain. This response may be a substantial confounder of experimental data and increase inter- and intra-animal variation ${ }^{18}$. To overcome these problems, and to assure continuous and stress-free administration of analgesia, depot formulations of analgesia for mice and rats have been developed ${ }^{19,20}$. Voluntary oral administration of analgesia is another promising approach that avoids the negative effects of handling. Several routes of oral administration have been described, such as mixing analgesics with flavored gelatine ${ }^{21}$, Nutella ${ }^{22}$, regular diet ${ }^{23}$ or drinking water ${ }^{24}$. These studies in mice and rats have shown that several analgesics are efficient when administered orally and voluntarily. Nevertheless, oral self-administration has been criticized as being less effective than subcutaneous treatment in rats $^{25}$. Reduced bioavailability caused by metabolizing of the drug before it reaches systemic circulation is a known obstacle in this administration route ${ }^{26}$. Moreover, latency to ingestion as well as the total amount ingested by the animals, especially during the resting phase, is difficult to anticipate and is clearly variable for each individual. Thus, voluntary ingestion protocols might be applicable only when pain is mild, or in combination with drug injections, at least during the resting phase of rodents ${ }^{24}$.

When surgical procedures are planned, analgesia has to be adjusted for the anesthetic protocol, especially if pre-emptive analgesia is applied. Pre-emptive analgesia is the administration of an analgesic drug before the nociceptive insult to reduce sensitization of the pain pathways. This procedure is widely recommended when pain is expected because many anesthesia agents, such as isoflurane, do not induce analgesia. Pre-emptive analgesia has the potential to be more effective than a similar analgesic treatment initiated after surgery and to reduce the required analgesia dose. Nevertheless, common analgesics have side effects that might hamper anesthetic or surgical procedures, such as respiratory depression or increased risk of bleeding. Current evidence concerning the beneficial effect of pre-emptive analgesia on chronic pain states is still sparse and further research is needed ${ }^{27}$.

\section{Side effects of opioid analgesics}

Opioids, which are used routinely in the treatment of laboratory rodents, are potent analgesics that exert their pharmacological effects by binding and activating specific opioid receptors that are widely distributed in the nervous system and gastrointestinal tract. The pain-relieving effect of opioid analgesics is induced by two mechanisms: inhibitory effects on pain transmission and emotional detachment from pain ${ }^{28-30}$. Given the abundant distribution and distinct receptor characteristics, side effects of opioids are diverse and include constipation, respiratory depression, nausea and urinary retention, as well as addiction, tolerance and hyperalgesia ${ }^{30}$.

Tolerance is characterized by a progressive lack of response to the drug that can be overcome by increasing the dose $\mathrm{e}^{31,32}$. Hyperalgesia is a central sensitization processes by which opioids sometimes increase rather than decrease pain ${ }^{31,32}$. In rodent studies, opioid-induced pain hypersensitivity has been observed after repeated or acute administration, and after high as well as low doses of opioids such as morphine, buprenorphine, tramadol or fentanyl ${ }^{31,33-36}$. Nevertheless, doubts have been raised regarding the actual significance of opioid-induced hyperalgesia in clinical settings using standard doses of opioids.

A topic of much discussion is the immune modulatory effects of some opioids. Immune modulation refers to stimuli that can alter immune function by affecting the generation, function and maturation of immune cells by several proposed mechanisms, including action on immunocytes as well as the hypothalamic-pituitaryadrenal (HPA) axis, sympathetic activity or central immune modulation. These mechanisms have been characterized in both humans and laboratory animals, and there are differences among opioids. Tramadol seems to have a weak upregulation effect, fentanyl and 
morphine appear to strongly downregulate the immune system, and buprenorphine causes only weak or no immunosuppression ${ }^{37,38}$. These effects should be taken into consideration when researchers are interested in immune responses. The effect of opioids on cancer development that was shown in animal studies has to be taken into account when planning pain treatment in oncological models. In mice, for example, tramadol seems to inhibit proliferation, migration and invasion of breast cancer cells ${ }^{39}$, whereas fentanyl inhibits tumor growth and cell invasion in colorectal cancer ${ }^{40}$. For morphine, tumor-enhancing effects after administration of daily morphine, as well as tumor and metastasis suppression, have been observed in mice ${ }^{41,42}$. In addition, it has been recognized that opioid receptors modulate inflammation, and reports are suggestive of both anti-inflammatory and pro-inflammatory effects ${ }^{29}$.

Endogenously released opioid peptides, as well as exogenously administered opioids, can have cardioprotective effects ${ }^{43}$. For example, in rodent ischemia reperfusion experiments, reduced infarct size was seen after preconditioning with fentanyl ${ }^{44}$.

In humans and animals, chronic opioid use alters endocrine function by inhibiting the hypothalamic-pituitary-gonadal (HPG) axis and possibly the HPA axis ${ }^{45}$. Negative effects of short-term treatment with common opioids, for example, buprenorphine, on reproductive parameters after embryo transfer are not known in mice ${ }^{46}$.

Although the effects listed above might be relevant only in experiments involving the affected systems, other side effects of opioids can affect the general condition of an animal, and might therefore be of interest for many research fields. Opioid-induced respiratory depression and other opioid-related respiratory responses are well-known side effects of opioid treatment and are caused by the activation of opioid receptors expressed in the respiratory centers of the brain stem ${ }^{47}$. These effects might lead to complications during anesthesia and the post-anesthetic recovery period. Conflicting results exist on whether opioids impair wound healing or not. Chronic morphine administration lengthens time to wound closure in rats and mice by suppressing angiogenesis, whereas improved wound healing has been demonstrated in the rat after topical application of fentanyl, hydromorphone and morphine ${ }^{48}$.

Reduction of food intake and body weight gain are well-known side effects of opioids such as buprenorphine in mice and rats ${ }^{19,49}$, and might be related to constipation and nausea. Opioid-induced constipation is a common effect of chronic opioid use. The mechanisms involve effects on the enteric nervous system that result in decreased intestinal fluid secretion and increased fluid absorption, as well as decreased motility of the small intestine and colon, leading to increased colonic transit time ${ }^{50}$. Opioids cause nausea and vomiting in humans. The pica behavior, also called allotriophagy or geophagy, is the rodent equivalent to the symptom of vomiting in other species. It involves eating non-nutritious substances, in most cases bedding or nesting material ${ }^{51,52}$. A single injection of buprenorphine, for example, is sufficient to induce this uncontrolled eating behavior, which can be life-threating. Although pica is regularly reported after buprenorphine administration, especially in rats, detailed information on its clinical course is lacking. When pica behavior occurs, a reduction in opioid dose, or replacement with a non-opioid drug, might be necessary. Opioids affect behavior distinctly. Behavioral side effects of buprenorphine in mice include circling, tiptoe gate, bent tail and an increase of activity that leads to a flatting of the circadian rhythmicity of the animals ${ }^{19}$. In contrast, in rats, buprenorphine has a sedating effect ${ }^{53}$.

\section{Side effects of NSAIDs and Coxibs}

Non-steroid anti-inflammatory drugs (NSAIDs) are a heterogeneous group of organic acids with analgesic, antipyretic, anti-inflammatory and platelet inhibitory actions. The main mechanism of action is inhibition of cyclooxygenase (COX) activity, with consequent inhibition of the synthesis of prostaglandins, which are involved as mediators of inflammation. Two enzymes are involved. COX 1, which is expressed in many cells and tissues, is important for normal homeostasis, whereas COX 2 is primarily and selectively induced by pro-inflammatory cytokines at the site of inflammation. It has been argued that selective COX 2 inhibitors, also known as Coxibs, might have reduced side effects, particularly causing less gastrointestinal toxicity. Nevertheless, treatment with Coxibs may carry an increased risk of adverse cardiovascular effects. Most cardiovascular and gastrointestinal side effects, and the renal toxicity of some NSAIDs and Coxibs, are associated with long-term use $\mathrm{e}^{30,54}$.

Although the administration of NSAIDs before surgery may have beneficial effects, such as reducing inflammation at the surgical site, minimizing amplification of nociceptive input and decreasing pain after surgery, NSAIDs are sometimes withheld because of their possible effect on platelet function and the consequent risk of perioperative (or gastrointestinal) hemorrhage ${ }^{54}$. Excessive perioperative hemorrhage may lead to longer surgical and anesthesia times and an increased risk of complications. However, not all NSAIDs irreversibly block platelet function. Platelets are vulnerable to COX 1 inhibition; thus, the perioperative administration of Coxibs may be a safer alternative to traditional NSAIDs in surgical procedures in which increased bleeding is a concern ${ }^{55}$.

Several studies have shown that NSAIDs, Coxibs and aspirin help to prevent cancer ${ }^{56}$. In particular, the effect of meloxicam has been described as inhibiting primary tumor growth in hepatocellular carcinoma or ovarian cancer mouse models ${ }^{57,58}$, as well as having an anti-metastatic effect in an orthotopic osteosarcoma mouse model ${ }^{59}$.

Prostaglandins also have a role in female reproductive processes. In mice, inhibition of COX2 activity has been shown to result in failure of ovulation, fertilization and implantation ${ }^{60,61}$. Thus, the use of NSAIDs might bear a pregnancy risk factor, suggesting that the use of NSAIDs could negatively affect reproductive outcomes in, for example, mouse embryo transfer. Nevertheless, several studies detected no detrimental effects, but rather showed beneficial effects, of NSAID analgesia for embryo transfer, for example, with flunixin or carprofen/buprenorphine treatment ${ }^{46,60}$.

The effects of analgesics on bone healing after trauma have been widely discussed. Inflammation, involving prostaglandin synthesis by COX2, is an essential step in fracture healing. Data from animal studies suggest that NSAIDs and Coxibs, which inhibit COX2 and the formation of prostaglandins, can impair fracture healing and delay union. Nevertheless, the results of clinical human studies and experimental animal studies on the effect of COX2 inhibition on fracture healing have been inconclusive and suggest that the effects are 
reversible, and depend on the timing, duration and dose of treatment ${ }^{55,62}$. COX2 inhibitors, despite being widely used in the shortterm treatment of fracture pain in humans, should be considered a potential risk factor for fracture healing, and should be replaced in animal experiments, such as in examinations in the early healing stage. A widely used alternative in these models is the opioid tramadol.

In comparison with bone healing, few studies have looked at the effect of NSAIDs or Coxibs on soft tissue healing. Results of animal studies examining tendon and ligament healing are contradictory ${ }^{63}$. In rats, for example, systemic use of diclofenac has an anti-proliferative effect, but has no effect on clinical wound healing ${ }^{64}$, whereas the postoperative use of NSAIDs can decrease the strength of anastomotic tissue and increase the risk of anastomotic leakage ${ }^{65}$.

\section{Side effects of antipyretic drugs}

Paracetamol (acetaminophen) and acetylsalicylic acid (aspirin) are so-called antipyretic drugs, and are commonly used to treat mild-to-moderate acute pain and fever. Antipyretics and NSAIDs share a common mechanism: the inhibition of COX1 and COX2. In contrast with NSAIDs, both drugs inhibit prostaglandin synthesis in the nervous system rather than acting only peripherally; this central action is also the basis of their antipyretic effects. Details of the mechanism of the analgesic action of paracetamol still need further elucidation ${ }^{30}$.

Side effects of acetylsalicylic acid include damage to the gastrointestinal mucosa, inhibition of platelet aggregation and, in high doses, a higher bleeding tendency as well as hepatoxicity. Unlike acetylsalicylic acid, paracetamol does not have a substantial effect on platelets or on inflammation, and thus might be the preferred non-opioid analgesic when surgical bleeding is an issue $^{66}$. It has no effect on gastric mucosal lining, and therefore cannot produce the gastric ulceration caused by many NSAIDs. Although paracetamol is safe in usual dosages, its use might bear the risk of liver dysfunction in sensitive patients ${ }^{30}$. If used in combination with opioids, antipyretics reduce opioid consumption, but this reduction seems insufficient to reduce opioid-induced adverse effects in humans ${ }^{67}$.

\section{Multimodal therapies and local anesthesia}

Despite the standard use of multimodal therapies in humans and large laboratory species, their use remains uncommon in small rodents ${ }^{2}$. Multimodal analgesia is a concept that involves different classes of analgesics and/or different sites of drug administration. Administering a combination of two or more drugs with well-established, possibly complementary, pharmacokinetics and mechanism of action has been shown to result in a synergistic or additive effect ${ }^{68}$. The potential advantages over monotherapy are maximization of analgesic effect and minimization of side effects, as reduced amounts of each drug are needed ${ }^{55}$. Nevertheless, combinations of drugs might also have additive side effects, for example, the combination of acetylsalicylic acid and NSAIDs escalates the effect on the gastrointestinal mucosa ${ }^{30}$. Thus, the side effect profile of all drugs has to be analyzed carefully. For rodents, several effective multimodal analgesia protocols have been described, with examples including tramadol-carprofen ${ }^{69}$ or fentanyltrazodone-paracetamol ${ }^{70}$.
Adjuvant analgesics (drugs that are not designed primarily for pain relief) that might potentiate the effects of analgesics include antidepressants, anticonvulsants, local anesthetics and steroids $s^{71,72}$. Local anesthetics are commonly used adjuvant analgesics in human patients or larger animal species. Combining long-acting local anesthesia in the wound area and non-opioid analgesics is a basic concept in human short-stay patients, and might reduce the need for systemic pain relief ${ }^{55}$. Local anesthesia can be administered via surface, infiltration or epidural analgesia, and substances include lidocaine or ropivacaine. In small rodents, local anesthesia can be used only as an improvement to analgesia, but cannot replace general anesthesia, as any manipulation can induce stress in the animal.

\section{Conclusion}

Pain is a major welfare issue in animal experiments, and must be treated and minimized for ethical reasons. In addition, unrelieved pain may have a substantial and poorly controllable effect on many organs and animal behavior, and has the potential to increase the variability of data. Untreated pain may affect complex behavioral traits such as circadian rhythmicity or decision making, attention and learning via motivational changes, may change sensory capacities of animals via allodynia and hyperalgesia, or affect many physiological and endocrine systems via HPA activation. Thus, adequate pain relief also has an important scientific and methodological dimension.

Analgesia is one of the many experimental interventions applied to laboratory animals, and everyone involved with in vivo experiments should be aware of their potential side effects. Nevertheless, if analgesia protocols are chosen with care, effects of analgesia are controllable and, to a certain extent, standardizable. If information on side effects of new analgesia protocols in specific experiments is missing, the inclusion of an analgesia control group might be advisable. The publications based on such applied approaches can give valuable insights for the scientific community working with laboratory animals. It should be noted that an important prerequisite for reproducible, standardized and accessible animal experiments is the proper and complete reporting of every analgesic intervention ${ }^{2}$.

Although standardized pain management protocols are highly appreciated, pain management needs continuous learning, and requires assessment and adjustment for individual animals, even for animals undergoing similar procedures. Standard, rule-of-thumb analgesic protocols are not always appropriate. In addition to promoting animal welfare, providing laboratory animals with optimal analgesia might also improve the clinical relevance of animal models, as customized pain relief protocols are more reflective of the medical treatment of human patients.

It should be kept in mind that any use of analgesia is a shot in the dark when not accompanied by a suitable pain assessment and monitoring plan. Score sheets help to formalize and standardize the assessment and constant monitoring of welfare and pain. Such monitoring tools facilitate the implementation of refinement measures such as analgesia to improve the welfare of animals used in research. Score sheets should deploy meaningful parameters, such as robust and specific signs of pain, and signs that measure the actual, specific effect on the system or organ targeted by the experimental manipulation, as well as more general welfare measures. 
In addition to classical clinical signs, such as physiological symptoms and outer appearance, there are also several ethological indicators of pain and reduced well-being that have been introduced for mice and rats in the last decade. Indicators should be tested for their sensitivity (the ability to correctly identify animals with pain) and specificity (the ability to identify those not suffering from pain $)^{73}$. Pain assessment should be frequent during expected pain peaks, for example, during the first $12 \mathrm{~h}$ to first days after surgery or during the late stage of painful progressing diseases. The mode of detection of residual pain and the threshold scores that will trigger any following action should be established in advance and be followed up by adequate remedial intervention (such as providing appropriate analgesia or termination of the procedure).

In summary, reproducibility and transparency are basic principles of science and prerequisites for the scientific and ethical justification of animal experimentation. Formalized, standardized and adequate pain monitoring and treatment regimens can easily be reported in publications and may contribute to better transparency and reproducibility. Thus, they may be able to increase scientific quality and reduce unnecessary suffering and animal numbers.

\section{ACKNOWLEDGMENTS}

The author thanks M. Arras for critical revision of the manuscript.

\section{COMPETING FINANCIAL INTERESTS}

The author declares no competing financial interests.

\section{Received 15 November 2016; accepted 30 December 2016}

Published online at http://www.nature.com/laban

1. Pain terms: a list with definitions and notes on usage. Recommended by the IASP Subcommittee on Taxonomy. Pain 6, 249 (1979).

2. Carbone, L. \& Austin, J. Pain and laboratory animals: publication practices for better data reproducibility and better animal welfare. PLOS ONE 11, e0155001 (2016).

3. Purves, D., Augustine, G., Fitzpatrick, D. \& Hall, W. Neuroscience, 4th edn. (Sinauer Associates, 2008).

4. Schwarz, C. et al. Mechanical load modulates the stimulatory effect of bmp2 in a rat nonunion model. Tissue Eng. Part A 19, 247-254 (2013).

5. Arras, M., Rettich, A., Cinelli, P., Kasermann, H.P. \& Burki, K. Assessment of post-laparotomy pain in laboratory mice by telemetric recording of heart rate and heart rate variability. BMC Vet. Res. 3, 16 (2007).

6. Henke, J. \& Erhardt, W. Schmerzmanagement beim Klein- und Heimtier (ENKE, Stuttgart, 2001).

7. Carstens, E. \& Moberg, G.P. Recognizing pain and distress in laboratory animals. ILAR J. 41, 62-71 (2000).

8. Page, G.G. The immune-suppressive effects of pain. Adv. Exp. Med. Biol. 521, 117-125 (2003).

9. Jirkof, P. et al. Assessment of postsurgical distress and pain in laboratory mice by nest complexity scoring. Lab. Anim. 47, 153-161 (2013).

10. Jirkof, P. et al. Burrowing behavior as an indicator of post-laparotomy pain in mice. Front. Behav. Neurosci. 4, 165 (2010).

11. Jirkof, P., Cesarovic, N., Rettich, A., Fleischmann, T. \& Arras, M. Individua housing of female mice: influence on postsurgical behavior and recovery. Lab. Anim. 46, 325-334 (2012).

12. Mogil, J.S. et al. Heritability of nociception I: responses of 11 inbred mouse strains on 12 measures of nociception. Pain 80, 67-82 (1999).

13. Mogil, J.S. The genetic mediation of individual differences in sensitivity to pain and its inhibition. Proc. Natl. Acad. Sci. USA 96, 7744-7751 (1999).

14. Davidson, S., Truong, H. \& Giesler, G.J. Jr. Quantitative analysis of spinothalamic tract neurons in adult and developing mouse. J. Comp. Neurol. 518, 3193-3204 (2010).

15. Bodnar, R.J. \& Kest, B. Sex differences in opioid analgesia, hyperalgesia, tolerance and withdrawal: central mechanisms of action and roles of gonadal hormones. Horm. Behav. 58, 72-81 (2010).
16. Collins, D., Reed, B., Zhang, Y. \& Kreek, M.J. Sex differences in responsiveness to the prescription opioid oxycodone in mice. Pharmacol. Biochem. Behav. 148, 99-105 (2016).

17. Cinelli, P., Rettich, A., Seifert, B., Burki, K. \& Arras, M. Comparative analysis and physiological impact of different tissue biopsy methodologies used for the genotyping of laboratory mice. Lab. Anim. 41, 174-184 (2007).

18. Moberg, G.P. When does stress become distress? Lab Anim. (NY) 28 , 22-26 (1999).

19. Jirkof, P., Tourvieille, A., Cinelli, P. \& Arras, M. Buprenorphine for pain relief in mice: repeated injections vs sustained-release depot formulation. Lab. Anim. 49, 177-187 (2015).

20. Foley, P.L., Liang, H. \& Crichlow, A.R. Evaluation of a sustained-release formulation of buprenorphine for analgesia in rats. J. Am. Assoc. Lab. Anim. Sci. 50, 198-204 (2011).

21. Liles, J.H., Flecknell, P.A., Roughan, J. \& Cruz-Madorran, I. Influence of oral buprenorphine, oral naltrexone or morphine on the effects of laparotomy in the rat. Lab. Anim. 32, 149-161 (1998).

22. Goldkuhl, R., Jacobsen, K.R., Kalliokoski, 0., Hau, J. \& Abelson, K.S. Plasma concentrations of corticosterone and buprenorphine in rats subjected to jugular vein catheterization. Lab. Anim. 44, 337-343 (2010).

23. Molina-Cimadevila, M.J. et al. Oral self-administration of buprenorphine in the diet for analgesia in mice. Lab. Anim. 48, 216-224 (2014).

24. Sauer, M., Fleischmann, T., Lipiski, M., Arrasa, M. \& Jirkof, P. Buprenorphine via drinking water and combined oral-injection protocols for pain relief in mice. Appl. Anim. Behav. Sci. 185, 103-112 (2016).

25. Thompson, A.C., DiPirro, J.M., Sylvester, A.R., Martin, L.B. \& Kristal, M.B. Lack of analgesic efficacy in female rats of the commonly recommended oral dose of buprenorphine. J. Am. Assoc. Lab. Anim. Sci. 45, 13-16 (2006).

26. Brewster, D., Humphrey, M.J. \& Mcleavy, M.A. The systemic bioavailability of buprenorphine by various routes of administration. J. Pharm. Pharmacol. 33, 500-506 (1981).

27. Dahl, J.B. Pre-emptive analgesia. Lancet 342, 561-562 (1993).

28. Williams, J.T. et al. Regulation of mu-opioid receptors: desensitization, phosphorylation, internalization, and tolerance. Pharmacol. Rev. 65, 223-254 (2013).

29. Sehgal, N., Smith, H.S. \& Manchikanti, L. Peripherally acting opioids and clinical implications for pain control. Pain Physician 14, 249-258 (2011).

30. Aronson, J. Meyler's Side Effects of Analgesics and Anti-inflammatory Drugs. 1st edn. (Elsevier, 2010).

31. Rivat, C. \& Ballantyne, J. The dark side of opioids in pain management: basic science explains clinical observation. PAIN Rep. 1, e570 (2016).

32. King, T., Ossipov, M.H., Vanderah, T.W., Porreca, F. \& Lai, J. Is paradoxical pain induced by sustained opioid exposure an underlying mechanism of opioid antinociceptive tolerance? Neurosignals 14, 194-205 (2005).

33. Wala, E.P. \& Holtman, J.R. Jr. Buprenorphine-induced hyperalgesia in the rat. Eur. J. Pharmacol. 651, 89-95 (2011).

34. Lyons, P.J., Rivosecchi, R.M., Nery, J.P. \& Kane-Gill, S.L. Fentanyl-induced hyperalgesia in acute pain management. J. Pain Palliat. Care Pharmacother. 29, 153-160 (2015).

35. Lee, S.H. et al. Tramadol induced paradoxical hyperalgesia. Pain Physician 16, 41-44 (2013)

36. Crain, S.M. \& Shen, K.F. Acute thermal hyperalgesia elicited by low-dose morphine in normal mice is blocked by ultra-low-dose naltrexone, unmasking potent opioid analgesia. Brain Res. 888, 75-82 (2001).

37. Sacerdote, P. Opioid-induced immunosuppression. Curr. Opin. Support. Palliat. Care 2, 14-18 (2008)

38. Al-Hashimi, M., Scott, S.W., Thompson, J.P. \& Lambert, D.G. Opioids and immune modulation: more questions than answers. Br. J. Anaesth. 111 80-88 (2013).

39. Xia, M. et al. Tramadol inhibits proliferation, migration and invasion via alpha2-adrenoceptor signaling in breast cancer cells. Eur. Rev. Med. Pharmacol. Sci. 20, 157-165 (2016).

40. Zhang, X.L., Chen, M.L. \& Zhou, S.L. Fentanyl inhibits proliferation and invasion of colorectal cancer via beta-catenin. Int. J. Clin. Exp. Pathol. 8, 227-235 (2015).

41. Sasamura, T. et al. Morphine analgesia suppresses tumor growth and metastasis in a mouse model of cancer pain produced by orthotopic tumor inoculation. Eur. J. Pharmacol. 441, 185-191 (2002). 
42. Bimonte, S. et al. Morphine promotes tumor angiogenesis and increases breast cancer progression. Biomed Res. Int. 2015, 161508 (2015).

43. Tanaka, K., Kersten, J.R. \& Riess, M.L. Opioid-induced cardioprotection. Curr. Pharm. Des. 20, 5696-5705 (2014)

44. Xu, Y.C. et al. kappa-Opioid receptors are involved in enhanced cardioprotection by combined fentanyl and limb remote ischemic postconditioning. J. Anesth. 29, 535-543 (2015).

45. Brennan, M.J. The effect of opioid therapy on endocrine function. Am. J. Med. 126 Suppl 1, S12-S18 (2013).

46. Goulding, D.R. et al. The effects of perioperative analgesia on litter size in Crl:CD1(ICR) mice undergoing embryo transfer. J. Am. Assoc. Lab. Anim. 49, 423-426 (2010).

47. van der Schier, R., Roozekrans, M., van Velzen, M., Dahan, A. \& Niesters, M. Opioid-induced respiratory depression: reversal by non-opioid drugs. F1000Prime Rep. 6, 79 (2014).

48. McIntyre, M.K., Clifford, J.L., Maani, C.V. \& Burmeister, D.M. Progress of clinical practice on the management of burn-associated pain: lessons from animal models. Burns 42, 1161-1172 (2016).

49. Bomzon, A. Are repeated doses of buprenorphine detrimental to postoperative recovery after laparotomy in rats? Comp. Med. 56, 114-118 (2006).

50. Webster, L.R., Camilleri, M. \& Finn, A. Opioid-induced constipation: rationale for the role of norbuprenorphine in buprenorphine-treated individuals. Subst. Abuse. Rehabil. 7, 81-86 (2016).

51. Clark, J.A., Myers, P.H., Goelz, M.F., Thigpen, J.E. \& Forsythe, D.B. Pica behavior associated with buprenorphine administration in the rat. Lab. Anim. Sci. 47, 300-303 (1997).

52. Takeda, N., Hasegawa, S., Morita, M. \& Matsunaga, T. Pica in rats is analogous to emesis: an animal-model in emesis research. Pharmacol. Biochem. Behav. 45, 817-821 (1993).

53. Johnson, R.A. Voluntary running-wheel activity, arterial blood gases, and thermal antinociception in rats after 3 buprenorphine formulations. J. Am. Assoc. Lab. Anim. Sci. 55, 306-311 (2016).

54. Mathiesen, 0. et al. Adverse effects of perioperative paracetamol, NSAIDs, glucocorticoids, gabapentinoids and their combinations: a topical review. Acta Anaesthesiol. Scand. 58, 1182-1198 (2014).

55. Wickerts, L., Warren Stomberg, M., Brattwall, M. \& Jakobsson, J. Coxibs: is there a benefit when compared to traditional non-selective NSAIDs in postoperative pain management? Minerva Anestesiol. 77, 1084-1098 (2011).

56. Umar, A., Steele, V.E., Menter, D.G. \& Hawk, E.T. Mechanisms of nonsteroidal anti-inflammatory drugs in cancer prevention. Semin. Oncol. 43, 65-77 (2016).

57. Xin, B., Yokoyama, Y., Shigeto, T., Futagami, M. \& Mizunuma, H. Inhibitory effect of meloxicam, a selective cyclooxygenase-2 inhibitor, and ciglitazone, a peroxisome proliferator-activated receptor gamma ligand, on the growth of human ovarian cancers. Cancer 110, 791-800 (2007).

58. Jiang, $X$. et al. Combining kallistatin gene therapy and meloxicam to treat hepatocellular carcinoma in mice. Cancer Sci. 100, 2226-2233 (2009).

59. Husmann, K. et al. Primary tumor growth in an orthotopic osteosarcoma mouse model is not influenced by analgesic treatment with buprenorphine and meloxicam. Lab. Anim. 49, 284-293 (2015).

60. Parker, J.M., Austin, J., Wilkerson, J. \& Carbone, L. Effects of multimodal analgesia on the success of mouse embryo transfer surgery. J. Am. Assoc. Lab. Anim. Sci. 50, 466-470 (2011).

61. Lim, H. et al. Multiple female reproductive failures in cyclooxygenase 2-deficient mice. Cell 91, 197-208 (1997).

62. Pountos, I., Georgouli, T., Calori, G.M. \& Giannoudis, P.V. Do nonsteroidal anti-inflammatory drugs affect bone healing? A critical analysis. ScientificWorldJournal 2012, 606404 (2012).

63. Chen, M.R. \& Dragoo, J.L. The effect of nonsteroidal anti-inflammatory drugs on tissue healing. Knee Surg. Sports Traumatol. Arthrosc. 21, 540-549 (2013).

64. Krischak, G.D., Augat, P., Claes, L., Kinzl, L. \& Beck, A. The effects of non-steroidal anti-inflammatory drug application on incisional wound healing in rats. J. Wound Care 16, 76-78 (2007)

65. Ji, C. et al. Effect of non-steroidal anti-inflammatory drugs on the increasing the incidence of colonic anastomosis in rats. Int. J. Clin. Exp. Pathol. 8, 6126-6134 (2015).

66. Smith, H.S. Perioperative intravenous acetaminophen and NSAIDs. Pain Med. 12, 961-981 (2011).

67. McNicol, E.D., Ferguson, M.C., Haroutounian, S., Carr, D.B. \& Schumann, R. Single dose intravenous paracetamol or intravenous propacetamol for postoperative pain. Cochrane Database Syst. Rev. (5); CD007126 (2016).

68. Schug, S.A. Combination analgesia in 2005: a rational approach: focus on paracetamol-tramadol. Clin. Rheumatol. 25 Suppl 1, S16-S21 (2006).

69. Cannon, C.Z., Kissling, G.E., Goulding, D.R., King-Herbert, A.P. \& Blankenship-Paris, T. Analgesic effects of tramadol, carprofen or multimodal analgesia in rats undergoing ventral laparotomy. Lab Anim. (NY) 40, 85-93 (2011).

70. Fernández-Dueñas, V. et al. Fentanyl-trazodone-paracetamol triple drug combination: multimodal analgesia in a mouse model of visceral pain. Pharmacol. Biochem. Behav. 98, 331-336 (2011).

71. Fishbain, D. Evidence-based data on pain relief with antidepressants. Ann. Med. 32, 305-316 (2000).

72. Mitra, R. \& Jones, S. Adjuvant analgesics in cancer pain: a review. Am. J. Hosp. Palliat. Care. 29, 70-79 (2012).

73. Golledge, H. \& Jirkof, P. Score sheets and analgesia. Lab. Anim. 50, 411-413 (2016). 